\title{
N-Acetyl Cysteine Loaded-Niosomes as a Mucolytic Agent for Acute Diseases and Respiratory Disorders
}

\author{
Mehdi Kamali $^{1(\mathbb{D})}$, Allahyar Noori ${ }^{2,3, * \mathbb{D}}$, Reza Sayyad Soufdoost ${ }^{4(\mathbb{D})}$ \\ Applied Biotechnology Research Center, Bqiyatallah University of Medical Sciences, Tehran, Iran \\ Research Center for Prevention of Oral and Dental Diseases, Baqiyatallah University of Medical Sciences, Tehran, Iran \\ Exercise Physiology Research Center, Life Style Institute, Baqiyatallah University of Medical Science, Tehran, Iran \\ Dentistry Research Institute, School of Dentistry, Shahed University, Tehran, Iran \\ Correspondence: allahyarnoori@gmail.com;
}

Scopus Author ID 57216968769

Received: 22.07.2020; Revised: 23.08.2020; Accepted: 25.08.2020; Published: 30.08.2020

\begin{abstract}
Today, there is a strong interest in the development of new drug delivery systems using an inactive carrier for pharmaceutics. In recent years, several drug delivery systems have been introduced, such as liposomes, niosomes, transfersomes, and pharmacosomes. N-acetylcysteine, as a mucolytic agent, is prescribed for acute diseases and respiratory disorders, flu, colds, and bronchitis to reduce mucus viscosity. In this study, niosomes n-acetyl cysteine was prepared with a hydration thin film layer method. Niosome formation was confirmed by light and electron microscope images. To determine the average size of niosomes, zeta seizer instruments were used. To evaluate the effects of the medicine on niosomes membrane composition, Fourier transforms infrared spectra were obtained. The best formulation for this study was the Span 60 formula with a 70:30 ratio. An increase in drug concentration was accompanied by a similar rise in niosome capacity. The average size of niosome with different formulations was listed. The release time of the drug was obtained. The FTIR spectroscopy of pure drug, niosome, and niosome with drugs were taken. When the cholesterol was increased from 30\% micromole to 50\% micromole, niosome average size was increased. The molar ratio 70: 30 of span and cholesterol was more appropriate for the preparation of niosome drug delivery. By adding distill phosphate and acetyl Tri-methyl ammonium bromide to niosomeal compounds, the average vesicle size and the loading percentage were increased.
\end{abstract}

Keywords: Niosomes; N-Acetyl Cysteine; Novel Drug Delivery Systems; Liposomes.

(C) 2020 by the authors. This article is an open-access article distributed under the terms and conditions of the Creative Commons Attribution (CC BY) license (https://creativecommons.org/licenses/by/4.0/).

\section{Introduction}

Novel drug delivery systems are applied for better control of kinetics, dynamics, nonspecific toxicity, and antigenicity to increase therapeutic efficacy [1-3]. The development of a novel drug delivery system using a passive carrier is rapidly expanding and improving. Restriction in the use of medications of different diseases has pushed for the creation of intelligent drug delivery systems. In a short time period, a large number of vesicular drug delivery systems were produced, such as liposomes, niosomes, transfersomes, and pharmacosomes [4]. Niosomes are self-assembled non-ionic surfactant vesicles with or without incorporation of cholesterol and other lipids and have a similar structure to liposomes. Niosomes have micro and nanostructures and are widely studied as an alternative to liposome [4-10]. Researchers have modified the drug's poor absorption by vesicle, such as niosomes [11]. Niosomes also can increase the loading of the drug in multiple environments [11]. The vesicle is made up of two layers of non-ionic surface-active agents with or without the 
incorporation of cholesterol. In other words, the non-ionic surfactant vesicles are called niosomes [4, 5, 12-14]. The self-assembly of surfactant monomers occurs during solvation [5].

The formation of vesicles needs non-ionic surfactants and organic solvents[4, 5]. In recent years, considerable research has been done on niosomes as drug carriers. Drug-loaded niosomes are applied to reduce drug side effects and increase therapeutic effects $[4,5,15]$. NAcetyl Cysteine drug as a mucolytic is prescribed in flu, colds, bronchitis, etc., to reduce the viscosity of mucus. N-Acetylcysteine reduces disulfide bonds of mucosal proteins. Also, the function of the drug as an antioxidant is increased cell survival [16-18]. The recommended dose for intramuscular administration of drugs is $1200 \mathrm{mg} /$ day $[19,20]$. The drug enters the general circulation and is degraded by the biological system. In addition, it is unable to cross the cell membrane barriers. Therefore, it can be removed quickly, lowering its efficacy [20, 21].

However, trapping of the drug in a carrier has the advantage of correcting the therapeutic effects by direct delivery into or the vicinity of the target cell population in addition to prolongation of its shelf life [3, 20]. The main purpose of an intelligent drug delivery system is to increase efficacy and reduce toxicity [3, 20]. Niosomes have overcome a few disadvantages of liposome drug carriers such as chemical instability, variable purity of phospholipids, and high prices. For example, due to high permeability, niosomes perform better than liposomes in skin drug delivery [21]. In this study, a thin film layer hydration method was used for niosomes preparation as a carrier of $\mathrm{N}$ - acetyl cysteine. The loading dose of $\mathrm{N}$-acetyl cysteine depends on the method and type of materials used as the carrier membrane [15]; a variety of simple formulations, combinations, and membrane ions were designed and investigated. This study sits as an introduction to the use of N-Acetyl cysteine-niosomes for the treatment of the chemical injury.

\section{Materials and Methods}

\subsection{Materials.}

The materials used in this study consisted of Span 60, 40, 20, and Tween 20, 40, isopropyl alcohol, chloroform, methanol, ethanol, methyl ammonium bromide, and cholesterol, all of which were obtained from Merck Company(Germany). N-Acetylcysteine and Decetyl Phosphate purchased from Aldrich Company (Germany).

\subsection{Preparation of thin-film layer.}

$60.29 \mathrm{mg}$ of span 60 and $23.16 \mathrm{mg}$ cholesterol were dissolved in $10 \mathrm{ml}$ of chloroform/methanol with a ratio of 2:1 in a round bottom flask. Using a rotary device under optimal conditions, the solvent was removed. After removing the solvent, a thin and uniform film was formed on the wall of the flask, which was placed at $60^{\circ} \mathrm{C}$ oven overnight. These Films were formed from 70 mole $\%$ of Span 60 and 30 mole \% of cholesterol. The Span 60 film formed using this method was termed Span 60 (70:30). In addition, $43.06 \mathrm{mg}$ of Span 60 and $38.6 \mathrm{mg}$ cholesterol were dissolved in $10 \mathrm{~mL}$ of chloroform/methanol (2: 1), and as mentioned, the solvent was removed. The film was uniformly formed on the walls of the flask, containing 50 mole \% of cholesterol and 50 mole \% of Span 60. Therefore the Span 60 film formed using this method was termed Span 60 (50:50). Moreover, the layer of the thin film was prepared from Span 40 and Span 20 using the same method. 


\subsection{Hydration of thin-film and encapsulation.}

$50 \mathrm{mg}$ of $\mathrm{N}$-Acetyl cysteine was dissolved in $10 \mathrm{~mL}$ of phosphate buffer saline (PBS, $\mathrm{PH}=7.4)$. The buffer and the flask containing the film were heated first in a rotary bath followed by water hydration using a rotary at a speed of $100 \mathrm{rpm}$ at $60^{\circ} \mathrm{C}$ for one hour. The end solution was sonicated with $25 \mathrm{~Hz}$ power for 2 minutes at $25{ }^{\circ} \mathrm{C}$. In addition to facilitating niosome formation, sonication facilitates the formation of regular shaped niosomes and prevents their aggregation. Thus niosome simple formulas containing $\mathrm{N}$-acetylcysteine were formed. Each film is composed according to table 1 formulas, With $10 \mathrm{ml}$ of phosphate buffer ( $\mathrm{pH} \mathrm{7.4)}$ containing $50 \mathrm{mg}$ of $\mathrm{N}$-acetylcysteine hydrated. According to table 1, cholesterol was formed by $30 \%$ and $50 \%$ with each of the types of spans and tweens. In each of the formulas, cholesterol participated once with a $30 \%$ micromole ratio and again with a $50 \%$ micromole ratio.

Table 1. Design of simple niosome formulation.

\begin{tabular}{|c|c|c|c|c|c|}
\hline \multirow[t]{2}{*}{ Formulae } & \multirow[t]{2}{*}{$\begin{array}{l}\text { Cholesterol } \\
(\% \cdot \mathbf{m M})\end{array}$} & \multicolumn{3}{|c|}{$\begin{array}{c}\text { Span } \\
(\% \text { ، } \mathbf{m M})\end{array}$} & \multirow{2}{*}{$\begin{array}{c}\begin{array}{c}\text { Tween } \\
(\% \cdot \mathrm{mM})\end{array} \\
\text { 40wT }\end{array}$} \\
\hline & & Sp60 & Sp40 & Sp20 & \\
\hline Span 60 (70-30) & 30 & 70 & & & \\
\hline Span $60(50-50)$ & 50 & 50 & & & \\
\hline Span $40(70-30)$ & 30 & & 70 & & \\
\hline Span $40(50-50)$ & 50 & & 50 & & \\
\hline Span 20 (70-30) & 30 & & & 70 & \\
\hline Span $20(50-50)$ & 50 & & & 50 & \\
\hline Tween $40(70-30)$ & 30 & & & & 70 \\
\hline Tween 40 (50-50) & 50 & & & & 50 \\
\hline
\end{tabular}

\subsection{Compound niosome preparation.}

In addition to the span, tween was used to compound niosome preparation, as seen in table 2. According to table 2, Span 60 was mixed with Tween 40 and 20 with equal ratios, and each of these mixtures was formed once with $50 \%$ micromole, and once again with $30 \%$ micromole, cholesterol was combined to form the niosome vesicles.

Table 2. Design of combinatorial formulation of niosomes.

\begin{tabular}{|c|c|c|c|c|c|}
\hline \multirow[t]{2}{*}{ Formulae } & \multirow{2}{*}{$\begin{array}{l}\text { Cholesterol } \\
(\% \cdot \mathbf{m M})\end{array}$} & \multicolumn{2}{|c|}{ Span ( mM, \% ) } & \multicolumn{2}{|c|}{$\begin{array}{c}\text { Tween } \\
(\% \text { ، } \mathbf{m M}) \\
\end{array}$} \\
\hline & & Sp60 & Sp40 & Tw40 & Tw20 \\
\hline Span 60+Tween 40 (70-30) & 30 & 35 & & 35 & \\
\hline Span 60+Tween $40(50-50)$ & 50 & 25 & & 25 & \\
\hline Span 60+Tween 20 (70-30) & 30 & 35 & & & 35 \\
\hline Span 60+Tween 20 (50-50) & 50 & 25 & & & 25 \\
\hline Span 40+Tween 40 (70-30) & 30 & & 35 & 35 & \\
\hline Span 40+Tween 40 (50-50) & 50 & & 25 & 25 & \\
\hline
\end{tabular}

\subsection{Preparation of niosome with an ionic membrane.}

For the preparation of niosomes with an ionic membrane, $10 \mathrm{ml}$ of negatively charged distilled phosphate or $5 \mathrm{ml}$ of positively charged cetyl trimethyl ammonium Bromide were mixed, as seen in Table 3. The process was the same as simple niosome preparation. In Table 3 , the positive and/or negative charges were added to the membrane of the simple and combined formulas of the spans by the tri-methyl ammonium bromide and distill phosphate, respectively. 


\begin{tabular}{|c|c|c|c|c|c|}
\hline \multirow[t]{2}{*}{ Formulae } & \multirow[t]{2}{*}{$\operatorname{Ion}(\mathbf{m M})$} & \multirow{2}{*}{$\begin{array}{l}\text { Cholesterol } \\
(\%, \mathrm{mM})\end{array}$} & \multicolumn{2}{|c|}{$\operatorname{Span}(\%, \mathbf{m M})$} & \multirow{2}{*}{$\begin{array}{l}\operatorname{Tween}(\%, \mathrm{mM}) \\
\text { Tw40 }\end{array}$} \\
\hline & & & Sp60 & Sp40 & \\
\hline Span 40 (70-30)+CTAB & 10 & 30 & & 70 & \\
\hline Span 40 (70-30)+DCP & 10 & 30 & & 70 & \\
\hline Span $60(70-30)+$ CTAB & 10 & 30 & 70 & & \\
\hline Span $60(70-30)+\mathrm{DCP}$ & 5 & 30 & 70 & & \\
\hline Span 40+Tween 40 (70-30)+CTAB & 10 & 30 & & 35 & 35 \\
\hline Span 40+Tween 40 (70-30)+DCP & 10 & 30 & & 35 & 35 \\
\hline Span 60+Tween 40 (70-30)+CTAB & 10 & 30 & 35 & & 35 \\
\hline Span 60+Tween 40 (70-30)+DCP & 5 & 30 & 35 & & 35 \\
\hline
\end{tabular}

\subsection{Isolation of unencapsulated drug from niosomes.}

For isolation of unloaded drugs, the emulsified solution was centrifuged at 14,000 rpm at $4{ }^{\circ} \mathrm{C}$ for $30 \mathrm{~min}$. The supernatant was collected, and its volume was measured. The precipitate was washed twice with buffer.

\subsection{Determining the percentage of the trapped drug.}

To determine the percentage of trapped drug in niosomes, a standard curve of the pure drug was plotted. For this purpose, a solution of N-Acetyl cysteine with a concentration of 5 $\mathrm{mg} / \mathrm{ml}$ in $\mathrm{ddH} 2 \mathrm{O}$ was prepared. $20 \mathrm{ml}$ was injected into the chromatograph, and the standard curve was plotted. Washed niosomes precipitate in each formula was dissolved in $2 \mathrm{ml}$ PBS ( $\mathrm{pH}=7.4)$. Chloroform was added. The mixture was stirred until an entirely transparent aqueous phase was obtained. The mixture was centrifuged at $10,000 \mathrm{rpm}$ for $10 \mathrm{~min}$ at $4^{\circ} \mathrm{C}$. Aqueous phase on the chloroform was separated completely, and its volume was measured. Here, the lipid membrane of niosomes was dissolved in chloroform, and $\mathrm{N}$-acetylcysteine entered the aqueous phase. $20 \mathrm{ml}$ of the aqueous phase was injected into the chromatograph device. The resulting curve was compared with the standard curve. The amount of drug in the aqueous phase was determined, and consequently, the loading percentage was calculated, which is in the following:

The amount of drug contained in $20 \mu \mathrm{l}$ of transparent aqueous phase $\times$ The total volume of the transparent aqueous phase/ Total drug used for loading

\subsection{Determining the capacity of niosomes.}

In order to optimize the amount encapsulation of $\mathrm{N}$-acetyl cysteine in niosomes, from the span 60 formula with a 70:30 ratio, 14 thin and uniform films were produced.

Table 4. Determining capacity of niosomes.

\begin{tabular}{l|c|c} 
Formulae & $\begin{array}{c}\text { Concentration } \\
\text { Mg/ml }\end{array}$ & $\begin{array}{c}\text { Niosome } \\
\text { weightg }\end{array}$ \\
\hline Sp60 (70-30) & 5 & $\mathbf{1 . 2 5}$ gr. \\
\hline Sp60 (70-30) & 5 & $\mathbf{1 . 2 4}$ gr. \\
\hline Sp60 (70-30) & 10 & $\mathbf{1 . 6}$ gr. \\
\hline Sp60 (70-30) & 15 & $\mathbf{1 . 1 7}$ gr. \\
\hline Sp60 (70-30) & 20 & $\mathbf{0 . 8 4}$ gr. \\
\hline Sp60 (70-30) & 25 & $\mathbf{1 . 3 1}$ gr. \\
\hline Sp60 (70-30) & 30 & $\mathbf{1 . 2 8}$ gr.
\end{tabular}

The loading capacity was measured. According to table 4, each formula was hydrated with $10 \mathrm{ml}$ deionized distilled water containing a known concentration of the drug. Free drugs were separated by centrifugation. Sediment weight (Niosomes) was measured after washing 
twice with phosphate buffer saline. By determining the drug in each concentration, niosomal drug loading percentage and capacity were determined (Table 4).

\subsection{Release of $\mathrm{N}$-acetyl cysteine from niosome.}

Three thin film layers of formula sp60 (70-30) formed on the flask walls. Each film was hydrated in $10 \mathrm{ml}$ of PBS (PH =7.4) containing $30 \mathrm{mg} \mathrm{N}$-Acetylcysteine. By determining the amount of drug in the supernatant of each formula, the average percentage of drug loading was calculated. The release of $\mathrm{N}$-acetyl cysteine was measured at six-time points; one, two, four, six, twelve, and twenty- four hours. Each niosome formula was washed twice with PBS (PH $=7.4$ ) and divided into 6 parts. $400 \mu \mathrm{l}$ of normal saline was added to each sediment in the tube, giving rise to three groups of six-part microtubes. Finally, microtubes were placed into a shaking incubator at $37^{\circ} \mathrm{C}$ and $150 \mathrm{rpm}$. Samples were assessed at time intervals of one, two, four, six, twelve, and twenty-four hours. The supernatant was separated at $4{ }^{\circ} \mathrm{C}, 10,000 \mathrm{rpm}$, and 10 minutes centrifugation. The average amount of drug release was determined at the specified time points.

\section{Results and Discussion}

\subsection{Light and electron microscope.}

To confirm the formation of niosome, light, and electron microscope images were used (Figure 1, A-E). Five microliters emulsion of the diluted niosome precipitate was placed on the slide and observed with the optical microscope. Niosomal vesicles were observed with a magnification of X40 and X100 light microscopes. The vesicles were spherical in shape. All of the designed formulas were observed with an optical microscope and photographed by a camera after hydration and loading the drug. Span 60 and cholesterol with ratio (70:30) were prepared drug carriers with a uniform average size (Figure 1, A to D). The 20 microliters of diluted niosome precipitate were placed on a wide aluminum foil and then placed inside an incubator $\left(45^{\circ} \mathrm{C}\right)$. A thin smear was prepared from the samples, and a thin layer of platinum was placed on it after $24 \mathrm{~h}$ and also evaluated by SEM (560 and 998 magnification). The vesicles were well visible, and the pores were created by the buffer vapors on the vesicle. So, the vesicles were formed, and some buffer was trapped with the drug. The contents of the niosome vesicles were dependent on the size and material of the membrane (Figure 1, E, and F).

\subsection{The average size of niosomes and zeta potential.}

To determine the average size of niosomes, the zeta seizer MALVERN Red British model was used. In table 5, the average size of niosome with different formulations is listed. The simple formulas had an average size of $650 \mathrm{~nm}$ to 1.5 micrometers. The Span 60 formula with $70 \%$ micromole of span and cholesterol with $30 \%$ micromole had a size of $750 \mathrm{~nm}$. But, But, its average size was reached to 1.5 micrometers when the cholesterol amount was raised from 30 to 50 micromoles percent. In Span 40, when the cholesterol ratio was raised from 30 to 50 micromoles percent, its average size was increased from $650 \mathrm{~nm}$ to 1 micrometer. In simple formulas, with an increase in cholesterol from 30 to 50 micromoles percent, the average size of the niosomes was almost doubled. The increase in cholesterol caused the doubled size of niosome. The created vesicles had the same $100 \%$ dispersion. The formed niosomes from 
compound formulas had an average size of 600 to $800 \mathrm{~nm}$, and their average sizes were not significantly increased with an increase in cholesterol from 30 to 50 micromoles percent. In addition, the dispersion size of the combined niosomes was different from 60 to 90 percent. The diameter of a simple niosomes with a negative charge membrane was about 1 micrometer. The increase in cholesterol had no significant effect on its size, but the niosomes membrane with a simple formula and a positive charge had more than 2 micrometers in diameter. A negative charge increased the diameter of the niosome, but a positive charge increased the diameter of the same niosome by more than twice. The effect of ions and the increase of cholesterol in the membrane of the combined niosomes were not significant. The formed niosomes from span had an average size of 450 to $550 \mathrm{~nm}$. In this study, the largest of created niosome was related to simple formulas with a positive charge membrane ( 2.5 micrometers in diameter).

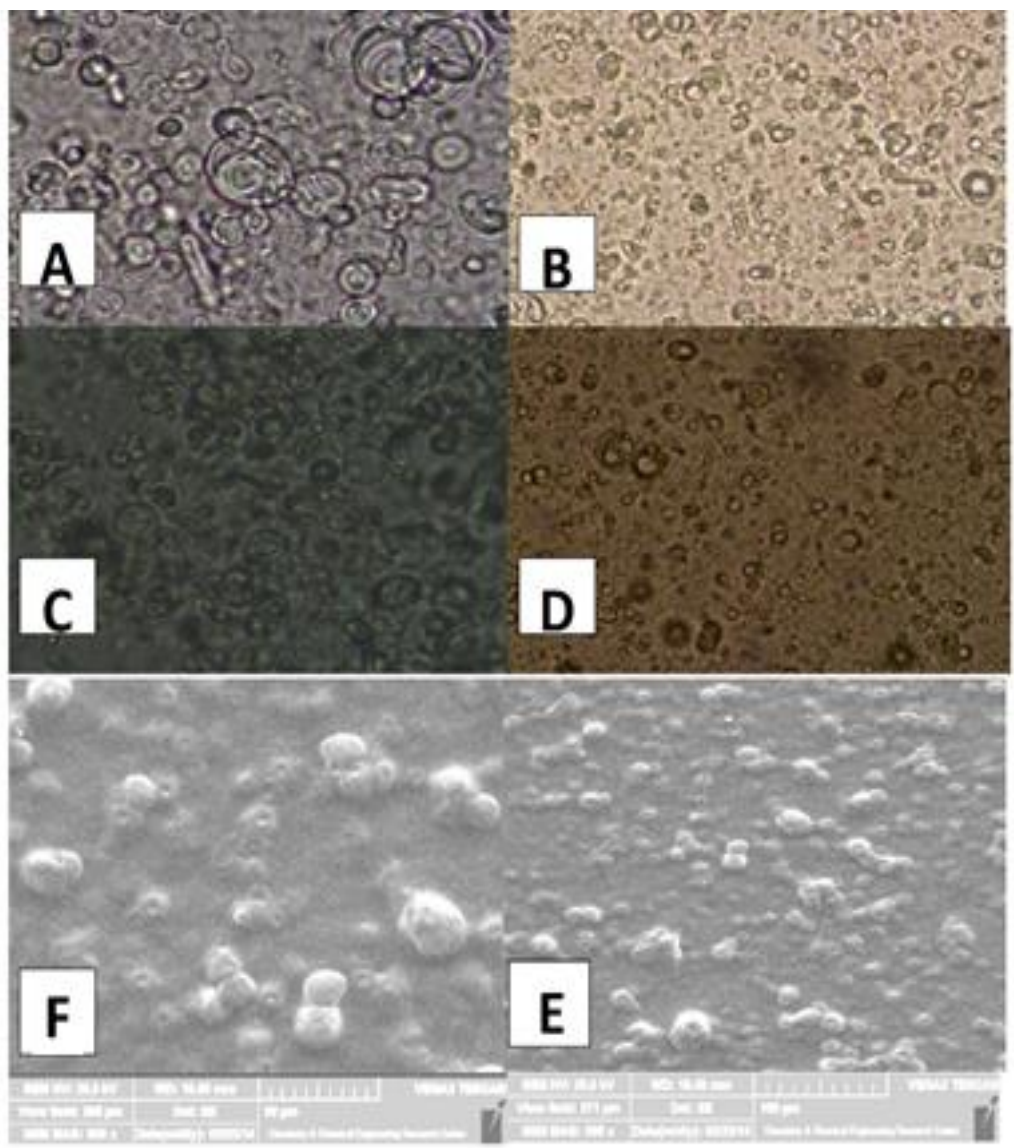

Figure 1. A. Niosomes image prepared from span 60 and tween 40 with a ratio of 50: 50, acetyl trimethyl ammonium bromide by light microscope with a magnification of $\times 100$. B. Niosomes image prepared from span 60 and tween 40 with a ratio of 30: 70 and distill phosphate by an optical microscope with a magnification of $\times$ 40. C. Niosomes image prepared from span 40 with a ratio of 30: 70 and distill phosphate by an optical microscope with a magnification $\times 40$. D. Niosomes image prepared from span 60 with a ratio of 50: 50 and $\times 100$ magnification by light microscopy. E. Niosomes image prepared from span 60 with a ratio of 30: 70 and distill phosphate by scanning electron microscope with a magnification of $\times 560$. F. Niosomes image prepared from span 60 with a ratio of $30: 70$ and distill phosphate by scanning electron microscope with a magnification of $\times 998$.

Table 5. Average size of niosome with different formulations.

\begin{tabular}{l|l|l|l} 
Formulae & Average $(\mathbf{d . n m})$ & Pdi & \% Intensity \\
\hline Sp60 (70-30) & $839 \pm 62$ & 0.230 & 100 \\
\hline Sp60 (50-50) & $1652 \pm 94$ & 0.428 & 100 \\
\hline Sp40 (70-30) & $691 \pm 47$ & 0.175 & 100 \\
\hline Sp40 (50-50) & $1004 \pm 72$ & 0.352 & 100 \\
\hline Sp40 +T40 (70-30) & $677 \pm 49$ & 0.452 & 81
\end{tabular}




\begin{tabular}{|c|c|c|c|}
\hline Formulae & Average(d.nm) & Pdi & $\%$ Intensity \\
\hline Sp40 +T40 (50-50) & $777 \pm 57$ & 0.277 & 94.2 \\
\hline Sp60 +T40 (70-30) & $672 \pm 51$ & 0.525 & 62 \\
\hline Sp60+T40 (50-50) & $839 \pm 43$ & 0.462 & 75 \\
\hline Sp60 (70-30) + CTAB & $2366 \pm 179$ & 0.652 & 85 \\
\hline Sp60 (50-50) + CTAB & $2357 \pm 186$ & 0.602 & 100 \\
\hline Sp60 (70-30) + DCP & $1180 \pm 77$ & 0.683 & 53 \\
\hline Sp60 (50-50) + DCP & $1314 \pm 84$ & 0.293 & 100 \\
\hline Sp40 (70-30) + CTAB & $2531 \pm 158$ & 0.678 & 90 \\
\hline Sp40 (50-50) + CTAB & $2366 \pm 120$ & 0.706 & 100 \\
\hline Sp40 (70-30) + DCP & $1037 \pm 65$ & 0.555 & 67 \\
\hline Sp40 (50-50) + DCP & $772 \pm 47$ & 0.529 & 55 \\
\hline Sp60 +T40 (70-30) +CTAB & $925 \pm 97$ & 0.551 & 91 \\
\hline Sp60+T40 (50-50) + CTAB & $1189 \pm 102$ & 0.579 & 63 \\
\hline Sp60 +T40 (70-30) +DCP & $937 \pm 52$ & 0.598 & 60 \\
\hline Sp60+T40 (50-50)+DCP & $738 \pm 69$ & 0.530 & 79 \\
\hline Sp40+T40 (70-30)+CTAB & $1224 \pm 97$ & 0.515 & 84 \\
\hline Sp40 +T40 (70-30) +DCP & $992 \pm 77$ & 0.685 & 55 \\
\hline Sp60(100), without cholesterol & $466 \pm 32$ & 0.747 & 70 \\
\hline $\begin{array}{ll}\text { Sp40+40(50-50), } & \text { without } \\
\text { cholesterol } & \end{array}$ & $549 \pm 55$ & 0.403 & 80 \\
\hline
\end{tabular}

\subsection{Zeta potentials of ionic membranes.}

Niosomes containing ions have surface charges. Therefore, the surface charge was determined by the zeta seizer. The values are shown in table 6 . The surface area of the vesicles was about 50 microvolts by adding 10 micromoles of phosphate or trimethyl ammonium bromide to simple and combined formulas.

Table 6. Zeta potentials of ionic membranes.

\begin{tabular}{l|c|c} 
Formulae & $\begin{array}{c}\text { Value charge } \\
(\boldsymbol{\mu m})\end{array}$ & $\begin{array}{c}\text { Zeta potentials } \\
(\mathbf{m V})\end{array}$ \\
\hline Sp40+CTAB & 10 & 52 \\
\hline Sp40+ DCP & 10 & -45 \\
\hline Sp60+ CTAB & 10 & 49 \\
\hline Sp60+ DCP & 5 & -15 \\
\hline Sp40+ T40+CTAB & 10 & 47 \\
\hline Sp60+ T40+ CTAB & 10 & 45 \\
\hline Sp60+ T40+ DCP & 5 & -19 \\
\hline Sp40+ T40+ DCP & 10 & -50
\end{tabular}

\subsection{Thin-film layer hydration and loading.}

Loading Percent of the drug in different niosome formulations is listed in table 7 . In niosomes, the amount of drug load was increased when the cholesterol ratio was reduced from 50 to 30 micromoles percent, although its diameter was reduced. The drug load was much lower in the combination membrane of niosomes than in simple membranes. The lowest drug load was in the formed niosomes of span without cholesterol. The newly formed niosomes had the maximum amount of $35 \%$ of drugs with a simple membrane and negative charge of span 60 and cholesterol with a ratio of 30 micromoles percent. The drug load was reduced to about $29 \%$ when the membrane load was changed to positive, while its diameter was more than doubled.

Table 7. Loading Percent of drug in different niosome formulations.

\begin{tabular}{l|c|c} 
Formula & Loading drug & Loading Percent $(\%)$ \\
\hline $60(70-30) p S$ & 12.95 & $\mathbf{2 5 . 9} \pm \mathbf{0 . 8 8}$ \\
\hline Sp60 (50-50) & 9.36 & $\mathbf{1 8 . 7} \pm \mathbf{0 . 6 8}$ \\
\hline Sp40 (70-30) & 11.33 & $\mathbf{2 2 . 7} \pm \mathbf{0 . 9 2}$
\end{tabular}




\begin{tabular}{l|c|c} 
Formula & Loading drug & Loading Percent $(\%)$ \\
\hline Sp40 $(50-50)$ & 5.61 & $\mathbf{1 1 . 2 1} \pm \mathbf{0 . 8 5}$ \\
\hline $60+\mathrm{T} 0(50-50) \mathrm{pS}$ & 7.04 & $\mathbf{1 4 . 1} \pm \mathbf{1 . 0 4}$ \\
\hline $40+\mathrm{T} 0(50-50) \mathrm{pS}$ & 3.39 & $\mathbf{6 . 8} \pm \mathbf{0 . 5 9}$ \\
\hline $60+\mathrm{T} 0(70-30) \mathrm{pS}$ & 5.59 & $\mathbf{1 1 . 1 9} \pm \mathbf{0 . 9 3}$ \\
\hline $40+\mathrm{T} 0(70-30) \mathrm{pS}$ & 5.36 & $\mathbf{1 0 . 7 3} \pm \mathbf{0 . 6 4}$ \\
\hline $60(70-30)+\mathrm{DCPpS}$ & 17.55 & $\mathbf{3 5 . 0 9} \pm \mathbf{1 . 1 1}$ \\
\hline $40(70-30)+\mathrm{DCPpS}$ & 10.4 & $\mathbf{2 0 . 8 3} \pm \mathbf{1 . 0 9}$ \\
\hline $60+\mathrm{T} 40(70-30)+\mathrm{DCP} \mathrm{pS}$ & 6.2 & $\mathbf{1 2 . 4 1} \pm \mathbf{1 . 4 5}$ \\
\hline $40+\mathrm{T} 40(70-30)+\mathrm{DCP} \mathrm{pS}$ & 9.1 & $\mathbf{1 8 . 0 8} \pm \mathbf{1 . 5 1}$ \\
\hline $60(70-30)+\mathrm{CTABpS}$ & 14.5 & $\mathbf{2 9 . 0} \pm \mathbf{1 . 2 8}$ \\
\hline $40(70-30)+\mathrm{CTABpS}$ & 14.42 & $\mathbf{2 8 . 8 4} \pm \mathbf{1 . 4 9}$ \\
\hline $60+\mathrm{T} 40(70-30)+\mathrm{CTABpS}$ & 14.09 & $\mathbf{2 8 . 1 8} \pm \mathbf{1 . 0 1}$ \\
\hline $40+\mathrm{T} 40(70-30)+\mathrm{CTABpS}$ & 7.48 & $\mathbf{1 4 . 4 9 \pm \mathbf { 1 . 6 3 }}$ \\
\hline Sp60 $(100)$ without cholesterol & & $\mathbf{2 . 3 9} \pm \mathbf{0 . 9 1}$ \\
\hline $\begin{array}{l}\text { Sp40+Tw40(50-50) } \\
\text { cholesterol }\end{array}$ & & $\mathbf{3 . 8 8} \pm \mathbf{1 . 2}$
\end{tabular}

Average $\pm \mathrm{SD}, \mathrm{n}=3$

\subsection{Niosome capacity.}

The concentration of $\mathrm{N}$ - Acetyl Cysteine during hydration was increased from $5 \mathrm{mg}$ to $30 \mathrm{mg}$ per $\mathrm{mL}$ (solvent: DI water with $\mathrm{pH}=7.4$ ). An increase in drug concentration was accompanied by a similar rise in niosome capacity. The results are listed in table 8 . According to table 8 , the amount of loading and the percentage of niomum capacity was increased with increasing drug concentrations. The maximum amount of drug was loaded at concentrations of 25 to $30 \mathrm{mg}$ of the drug per $\mathrm{mL}$, and the percentage of prepared niosome capacity with Span 60 formula was 6 percent. The release of niosomeal drugs during 24 hours is shown in table 9 . According to table 9 and figure 2, more than $50 \%$ of the drug was released during $6 \mathrm{~h}$. In $24 \mathrm{~h}$, more than $70 \%$ of the drug was released.

Table 8. Determining niosome capacity.

\begin{tabular}{l|c|c|c|c} 
Formulae & $\begin{array}{c}\text { Concentration } \\
(\mathbf{m g} / \mathbf{m l})\end{array}$ & $\begin{array}{c}\text { Niosome } \\
\text { weight(gr) }\end{array}$ & $\begin{array}{c}\text { Amount of } \\
\text { loaded drug(mg) }\end{array}$ & $\begin{array}{c}\text { Capacity } \\
(\mathbf{\%})\end{array}$ \\
\hline Sp60(70-30) & 5 & 1.25 & 12.82 & $\mathbf{1 . 0 5 6}$ \\
\hline Sp60(70-30) & 5 & 1.24 & 13.47 & $\mathbf{1 . 0 9}$ \\
\hline Sp60(70-30) & 10 & 1.6 & 20.36 & $\mathbf{1 . 2 7}$ \\
\hline Sp60(70-30) & 15 & 1.17 & 24.12 & $\mathbf{2 . 0 6}$ \\
\hline Sp60(70-30)+DCP & 20 & 0.84 & 34.87 & $\mathbf{4 . 1 5}$ \\
\hline Sp60(70-30) & 25 & 1.31 & 75.26 & $\mathbf{5 . 7 5}$ \\
\hline Sp60(70-30) & 30 & 1.28 & 74.59 & $\mathbf{5 . 8 3}$
\end{tabular}

Table 9. The time of release assessment (hours).

\begin{tabular}{l|c|c|c} 
Formulae & Amount of drug & The time of release & Amount of \\
in niosomes & assessment (hours) & release \\
\hline Sp60 (70-30) & 6.82 mg average & 1 & $\mathbf{0 . 1 2 6} \pm \mathbf{0 . 0 4}$ \\
\cline { 3 - 4 } & of drug in three & 2 & $\mathbf{0 . 3 1 3} \pm \mathbf{0 . 0 5}$ \\
\cline { 3 - 4 } & formulae & 4 & $\mathbf{0 . 4 8 1} \pm \mathbf{0 . 0 3}$ \\
\cline { 3 - 4 } & & 6 & $\mathbf{0 . 5 6} \pm \mathbf{0 . 0 7 4}$ \\
\cline { 3 - 4 } & & 12 & $\mathbf{0 . 6 5 4} \pm \mathbf{0 . 0 8}$ \\
\cline { 3 - 4 } & & 24 & $\mathbf{0 . 7 4 4} \pm \mathbf{0 . 1 1}$
\end{tabular}

Average $\pm \mathrm{SD}, \mathrm{n}=3$

According to the results table 10, the niosome with $800 \mathrm{~nm}$ diameter was created and traped $26 \%$ of the drug from the span 60 formula (30:70). When the amount of cholesterol was increased to $50 \mu \mathrm{mol}$, its volume was doubled, but the percentage of drug load was decreased to $19 \%$. In combination formulations, the diameter of the niosome was from 600 to 800 nanometers, and the drug load was from 7 to 14 percent. 


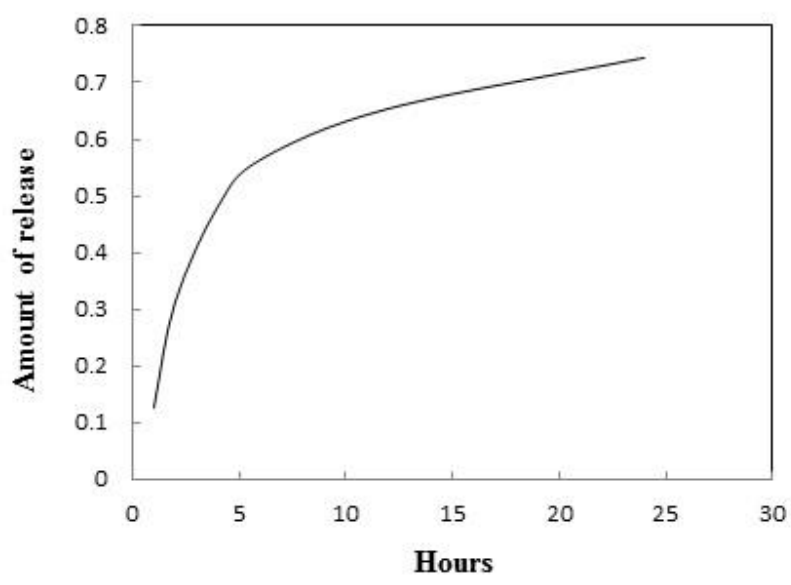

Figure 2. N-Acetyl cysteine was released at $24 \mathrm{hr}$.

Adding DCP to simple niosome formulas increased the diameter of the niosome to 1 micrometer and trapped $35 \%$ of the drug. When the same formula was found to be positive with the addition of $\mathrm{CTAB}$, the diameter of the niosome was more than double, but the drug load was $29 \%$, less than the niosome with a diameter of 1 micrometer. The achieved niosome with cholesterol-free from span had 400 to 500 nanometers in diameter. The smallest niosome was formed from cholesterol-free span formulation and loaded 2 until $4 \%$ of the drug.

Table 10. The relation between niosomal compounds with average seize and loading percent drug in niosome.

\begin{tabular}{l|c|c} 
Formulae & Average size $(\mathbf{d . n m})$ & Loading percent \\
\hline Sp60(70-30) & $839 \pm 62$ & $25.9 \pm 0.88 \%$ \\
Sp60(50-50) & $1652 \pm 94$ & $18.7 \pm 0.68 \%$ \\
\hline Sp40(70-30) & $691 \pm 47$ & $22.7 \pm 0.92 \%$ \\
Sp40(50-50) & $1004 \pm 72$ & $11.21 \pm 0.85 \%$ \\
\hline Sp40 +T40 (70-30) & $677 \pm 49$ & $10.73 \pm 0.64 \%$ \\
Sp40+T40 (50-50) & $777 \pm 57$ & $6.8 \pm 0.59 \%$ \\
\hline Sp60 +T40 (70-30) & $672 \pm 51$ & $14.1 \pm 1.04 \%$ \\
Sp60+T40 (50-50) & $839 \pm 43$ & $11.19 \pm 1.04 \%$ \\
\hline Sp60(70-30)+DCP & $1180 \pm 77$ & $35.09 \pm 1.11 \%$ \\
\hline Sp 40 (70-30)+DCP & $1037 \pm 65$ & $20.83 \pm 1.09 \%$ \\
\hline Sp60 (70-30)+CTABS & $2366 \pm 179$ & $29.0 \pm 1.28 \%$ \\
\hline Sp 40 (70-30)+CTAB & $2531 \pm 158$ & $28.84 \pm 1.49 \%$ \\
& & \\
Sp60(100) & $466 \pm 32$ & $2.39 \pm 0.91 \%$ \\
(without cholesterol & $549 \pm 55$ & $3.88 \pm 1.2 \%$ \\
Sp40+T40(50-50) without & & \\
cholesterol)( & &
\end{tabular}

Average $\pm \mathrm{SD}, \mathrm{n}=3$

\subsection{Spectrum of FT-IR.}

To investigate the effect of the N-Acetylcysteine on niosome membrane, Fourier transforms infrared (FTIR) spectra were obtained. For this reason, the spectra of various formulations of Span 60 (70:30) with and without drug were obtained separately. The FTIR spectroscopy of pure drug, niosome, and niosome containing drugs were taken. There were peaks of absorption areas in niosome without medication such as the alcohol-acting groups (3783), the ester-factor group (3373), and the hydrocarbon-acting group (2920, 2856). The peaks of niosome's acting groups were not reacted with the drug's acting groups. The drug was inserted into the niosome cavity without reacting with the agents of the niosome membrane (Figures 3, 4, and 5). 


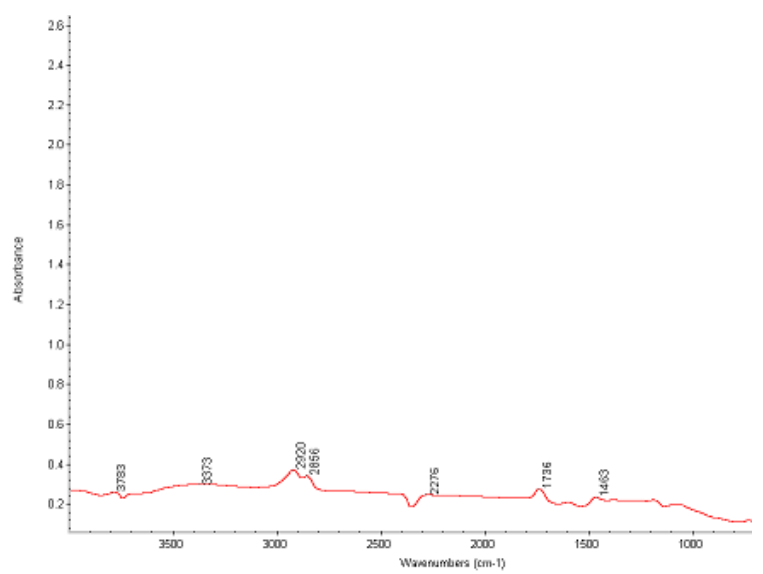

Figure 3. N-acetylcysteine Fourier transform infrared spectrum.

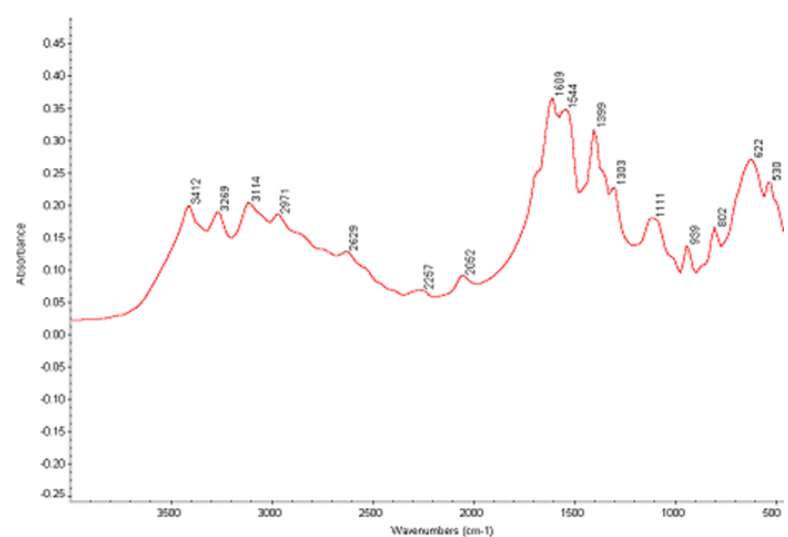

Figure 4. N-acetylcysteine Fourier transform infrared spectrum.

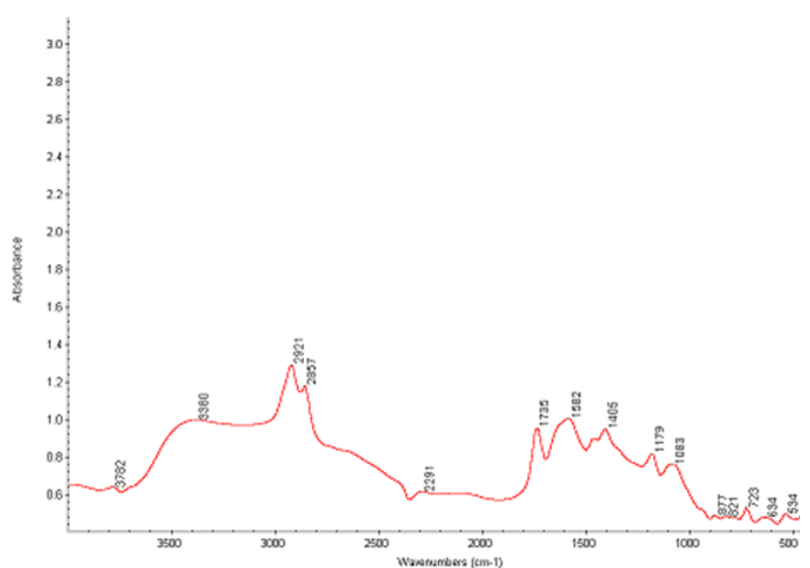

Figure 5. FT-IR spectrum of niosomes containing N-acetylcysteine.

\subsection{Discussion.}

As seen in table 10, niosome without cholesterol has a mean size of $500 \mathrm{~nm}$ and $3.2 \%$ loading. The lowest amount of loading and the smallest mean size of the niosomes are in this study. With the addition of $30 \%$ cholesterol micromole in niosome formula, the average size and the loading percentage increased. When the cholesterol increased from $30 \%$ micromole to $50 \%$ micromole, niosome average size increased. However, the loading percentage decreased. Mo'azeni et al. have reached similar conclusions [22]. On the other hand, Candasamy et al. have reported that cholesterol increases the hardness of niosome, not size [23]. In contrast to our finding, Pula Chandov has reported that by increasing in the niosome average size, The percentage of drug-loaded was increased [2]. 
However, the present study showed that by increasing the enclosed volume, the loading decreases. $30 \%$ cholesterol micromole incorporating niosome membrane could well cover the pores between Tween or Span chains in the structure, therefore lowering the drug leaking out of the vesicles. When the amount of cholesterol reached $50 \%$ micromole in the membrane of niosomes, disruption in the serial placement of Tween or Span chains occurs in niosome membrane structure. In other words, an increase in cholesterol side-chain of vesicles resulted in enlarging the size of the vesicles. In addition, it created pores in the membrane and favoring drug leakage, especially when a centrifuge was used to separate the free drug. Thus, by increasing the enclosed volume and subsequently increase in the average size of niosomee, drug leakage increases, and percent drug load decreases. Results show that the molar ratio 70: 30 of span and cholesterol is somehow more appropriate for the preparation of niosome drug delivery. By adding distill phosphate and Acetyl Tri-methyl ammonium bromide to niosomeal compounds, the average vesicle size and the loading percentage were both increased. Increased zeta potential of the electric double layer in between nisome's layers and the electrical repulsion between the layers may explain the phenomenon. This finding is in accordance with the findings of Ruckmany et al., and Mo'azeni et al. It is noteworthy that the percentage of loaded drug in the formulation composed of 30 mole $\%$ cholesterol was significantly higher. The capacity of niosomes is an important factor to consider for drug delivery. With an increase in drug concentration in the buffer (Table 8), niosome loading capacity increased. However, the negative charge of Decetyl Phosphate in the niosome membrane has led to an increase in the slope of the capacity angle in comparison to other drug concentrations.

\section{Conclusions}

Thus, it improves the drug load and increases the capacity of the niosome. Therefore, the formula Sp60 (70-30) + DCP in this study is better than other formulations for drug delivery. According to the diagram, the peak of the drug release occurred within 6 hours of niosome loading. This is not a good result. The small size of $\mathrm{N}$-acetylcystein $(163 \mathrm{D})$ can be a major factor in the rapid delivery. The rest of the loaded drug was released slowly after 6 hours. The absorption regions of factor groups in niosomes containing $\mathrm{N}$-acetyl cysteine are the same as drug and carrier. This means a new bond or functional group is not created in niosome with drugs, which had an infrared absorption spectrum. Therefore, $\mathrm{N}$-acetylcysteine has no chemical interaction with niosome compounds, but the drug is located within the niosome cavity.

\section{Funding}

This research received no external funding.

\section{Acknowledgments}

The authors would like to thank the Bqiyatallah University of Medical Sciences, Tehran, Iran.

\section{Conflicts of Interest}

The authors declare no conflict of interest.

\section{References}

1. Heidi, M.; Yun Seok, R.; Xiao, W. Nanomedicine in pulmonary delivery. Int J Nanomedicine 2009, 4, 299319, https://doi.org/10.2147/ijn.s4937. 
2. Chandu, V.; Arunachalam, A.; Jeganath, S.; Yamini, K.; Tharangini, K.; Chaitanya, G. Niosomes: a novel drug delivery system. Int J Novel Trends Pharm Sci 2012, 2, 25-31.

3. Smola, M.; Vandamme, T.; Sokolowski, A. Nanocarriers as pulmonary drug delivery systems to treat and to diagnose respiratory and non respiratory diseases. Int J Nanomedicine 2008, 3, 1-19.

4. Abhinav, K.; Lal, P.; Amit, J.; Wishwabhan, S. Review on niosomes as novel drug delivery system. Int Res J Pharm 2011, 2, 61-65.

5. Keservani, R.; Sharma, A.; Ayaz, M.; Kesharwani, R. Novel drug delivery system for the vesicular delivery of drug by the niosomes. Inte J Control Release 2011, 1, 1-8.

6. Pereira, M.C.; Pianella, M.; Wei, D.; Moshnikova, A.; Marianecci, C.; Carafa, M.; Andreev, O.A.; Reshetnyak, Y.K. pH-sensitive pHLIP((R)) coated niosomes. Mol Membr Biol 2016, 33, 51-63, https://doi.org/10.1080/09687688.2017.1342969.

7. Onochie, I.; Nwakile, C.; Umeyor, C.; Uronnachi, E.; Osonwa, U.; Attama, A.; Esimone, C. Formulation and Evaluation of Niosomes of Benzyl Penicillin. J Appl Pharm 2013, 3, 66-71.

8. Mansour, H.; Rhee, Y.; Wu, X. Niosomes: a novel trend of drug delivery. European j biomed pharm 2017, 4, 436-442.

9. Ruckmani, K.; Sankar, V. Formulation and optimization of Zidovudine niosomes. AAPS PharmSciTech 2010, 11, 1119-27, https://doi.org/10.1208/s12249-010-9480-2.

10. Yoshioka, T.; Sternberg, B.; Florence, A. Preparation and properties of vesicles (niosomes) of sorbitan monoesters (Span 20, 40, 60 and 80) and a sorbitan triester (Span 85). Int J Pharm 1994, 105, 1-6, https://doi.org/10.1016/0378-5173(94)90228-3.

11. Tarekegn, A.; Palani, S.; Zacharia, A.; Ayenew, Z.; Joseph, N. Niosomes in targeted drug delivery: some recent advances. In J Pharm Sci Res 2010, 1, 1-8, https://doi.org/10.13040/IJPSR.0975-8232.1(9-S).1-8.

12. Marianecci, C.; Di Marzio, L.; Rinaldi, F.; Celia, C.; Paolino, D.; Alhaique, F.; Esposito, S.; Carafa, M. Niosomes from 80s to Present: The state of the art. Adv Colloid Interfac 2014, 205, 187-206, https://doi.org/10.1016/j.cis.2013.11.018.

13. Moghassemi, S.; Hadjizadeh, A. Nano-niosomes as nanoscale drug delivery systems: an illustrated review. J Control Release 2014, 185, 22-36, https://doi.org/10.1016/j.jconrel.2014.04.015.

14. Obeid, M.A.; Gebril, A.M.; Tate, R.J.; Mullen, A.B.; Ferro, V.A. Comparison of the physical characteristics of monodisperse non-ionic surfactant vesicles (NISV) prepared using different manufacturing methods. Int J Pharm 2017, 521, 54-60, https://doi.org/10.1016/j.ijpharm.2017.02.007.

15. Sudhamani, T.P.N.; Radhakrishnan, M. Proniosomes - A Promising Drug Carriers. Int J Pharmtech Res 2010, 2, 1446-54.

16. Sadowska, A.; Verbraecken, J.; Darquennes, K.; De Backer, W. Role of N-acetylcysteine in the management of COPD. Int J Chron Obstruct Pulmon Dis 2006, 1, 425-34.

17. Sansone, R.; Sansone, L. Getting a Knack for NAC: N-Acetyl-Cysteine. Innov Clin Neurosci 2011, 8, 10-4.

18. Sadowska, A. N-Acetylcysteine mucolysis in the management of chronic obstructive pulmonary disease. Ther Adv Respir Dis 2012, 6, 127-35, https://doi.org/10.1177/1753465812437563.

19. Arefi, M.; Behnoush, B.; Pouraziz, H.; Yousefinejad, V. Comparison of oral and intravenous Nacetylcysteine administration in the treatment of acetaminophen poisoning. Sci J of Kurdistan Uni of Med Sci 2013, 18, 36-43.

20. Mitsopoulos, P.; Suntres, Z.E. Protective Effects of Liposomal N-Acetylcysteine against Paraquat-Induced Cytotoxicity and Gene Expression. J Toxicol 2011, 2011, https://doi.org/10.1155/2011/808967.

21. Lohumi, A. A Novel Drug Delivery System: Niosomes Review. J. drug deliv. ther 2012, 2, 129-135, https://doi.org/10.22270/jddt.v2i5.274.

22. Moazeni, E.; Gilani, K.; Sotoudegan, F.; Pardakhty, A.; Najafabadi, A.R.; Ghalandari, R.; Fazeli, M.R.; Jamalifar, H. Formulation and in vitro evaluation of ciprofloxacin containing niosomes for pulmonary delivery. J Microencapsul 2010, 27, 618-27, https://doi.org/10.3109/02652048.2010.506579.

23. Ruckmani, K. Formulation and Optimization of Zidovudine Niosomes. AAPS PharmSciTech 2010, 11, 1119-1127, https://doi.org/10.1208/s12249-010-9480-2. 